Document downloaded from:

http://hdl.handle.net/10251/52877

This paper must be cited as:

Guijarro Coloma, LA.; Pla, V.; Vidal Catalá, JR.; Martínez Bauset, J. (2015). Search engine and content providers: neutrality, competition and integration. Transactions on Emerging Telecommunications Technologies. 26(2):164-178. doi:10.1002/ett.2827.

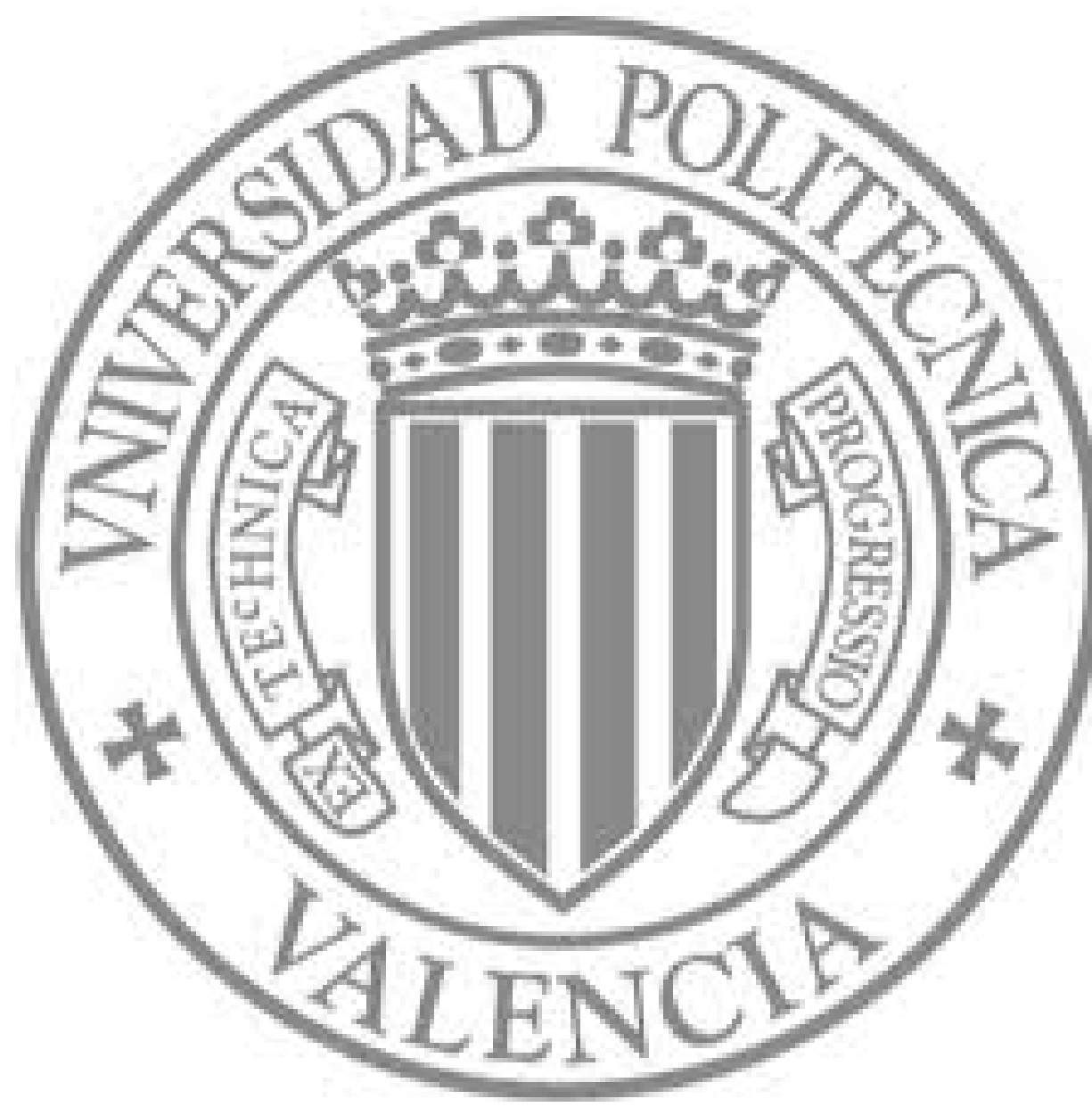

The final publication is available at

http://dx.doi.org/10.1002/ett.2827

Copyright John Wiley \& Sons 


\title{
Search engine and content providers: neutrality, competition and integration
}

\author{
Luis Guijarro*, Vicent Pla, Jose R. Vidal, Jorge Martinez-Bauset \\ Universitat Politècnica de València, Camino de Vera, s/n. 46022 Valencia, Spain
}

\begin{abstract}
In recent years, there has been a rising concern about the policy of major search engines. The concern comes from search bias, which refers to the ranking of the results of a keyword search on the basis of some other principle than the sheer relevance. This search bias is also named search non-neutrality. In this paper, we analyze one non-neutral behavior, that is, a behavior that results in a search bias: the payment by content providers to the search engine (aka. side payment) in order to improve the chances to be located and accessed by a user. A game theory-based model is presented where a search engine and two content providers interact strategically, while the aggregated behavior of users is modeled by a demand function. The utility of each stakeholder (i.e., the users, the search engine, and each content provider) when the search engine is engaged in such a non-neutral behavior is compared with that of the neutral case, when no such side payment is present. Additionally, the paper analyzes the organization of such an industry, specifically, the search engine and content providers incentives for a partial and full merger with the content providers, and the effects of each organization on the users. This paper concludes by identifying the circumstances under which the search bias, on the one hand, and the integration, on the other, will effectively result in the users being harmed. This eventual harmful situation will provide a rationale for regulatory measures to be adopted.
\end{abstract}

\section{* Correspondence}

Departamento de Comunicaciones. Universitat Politècnica de València, Camino de Vera, s/n. 46022 Valencia, Spain. E-mail: Iguijar@dcom.upv.es

\section{INTRODUCTION}

In recent years there has been an increasing concern about Google policy, in terms of its search activities, implying the eventuality that this role should be regulated by government authorities. This is the aim of "search engine neutrality" advocates. The concern is about search engine bias, a term used to describe the activities of a search engine exercising its editorial discretion in a manner that advantages its own or affiliated content, which could favor some content wishing to pay to be better ranked, or which could disadvantage rivals. Search bias therefore refers to rankings based on some principle other than automated relevance for users. Within this paper, we focus on this concern, shown by some regulators on those search rankings that benefit the host search engine: for example the European Commission justified its inquiry into Google by a need to investigate if there is a conduct potentially "lowering the ranking of unpaid results" relatively to paid advertisements even in the regular, also called organic, results, as opposed to the sponsored links clearly declared (and presented as such to users) to make money [1].

Search neutrality advocates need, however, to demonstrate that there is a problem necessitating any of the various prescribed remedies, such as the application of standard merger analysis under the antitrust laws and even 
the creation of a government-sponsored "public option" for searches [2].

Problems cited by search neutrality advocates may be broadly classified in two groups: competition law and antitrust problems arising from "non-objective" search results, and social and cultural issues arising from the use that consumers may make with "non-objective" results. This paper is focused on the former group.

As regards the potential competitive harm from search bias, one argument is based on the "essential facilities" doctrine [3] and stresses that popular search engines, Google for instance, act as a "bottleneck" to access of websites by consumers. By using its power to determine which end websites are reached or not, a search engine can effectively exclude nascent websites from both advertising and sales revenue. Another, but related, argument claims that a search engine disadvantages its content rivals by raising their (awareness) costs relatively to its own. By directing search traffic to its own products-e.g., its mail, calendar, and marketplace platforms - a search engine would effectively discriminate against rivals and force those rivals into more expensive substitute distributions channels [4]. The question remains whether a search engine's use of its search algorithm to direct traffic to itself harms competition and consumers [5].

This paper aims to shed light on the issue of the search bias. Specifically, it focuses on the analysis of the harm that an apparently non-neutral behavior by a search engine may have on content providers and users. The non-neutral behavior we specifically consider here consists in charging a side payment to the content providers in exchange for better search results. Additionally, the paper analyzes the organization of such an industry, specifically, the search engine and content providers incentives for partial and full integration. While the issue of network neutrality has recently been the topic of a very active debate and extensive literature (see [6] and references herein), this paper is to our knowledge one of the few ones dealing with a mathematical modeling and analysis of search neutrality, though this issue may be critical as we have just highlighted above.

Note that we have defined search neutrality by drawing parallelisms with the definition of network neutrality. Net neutrality means that Internet Service providers (ISPs) should charge consumers only once for Internet access, should not favor one content provider over another, and should not charge content providers serviced by competitors for sending information over broadband lines to end users-no matter how much bandwidth that content uses [7]. The debate around net neutrality arose when ISPs complained that major content providers had their traffic flowing through ISP networks-which was resource consuming — but were only paying an access fee to their servicing ISPs. ISPs then threatened to ask side payments to content providers [8]. In a similar fashion, as motivated in the previous paragraphs and for the purposes of our analysis, we define search neutrality as the provision of a search service to the users where the search engine does not request any side payment from the content providers.

This paper is structured as follows. Next section describes the scenario to be modeled and presents the details of the baseline (neutral) model, which comprises a search engine without a search bias, and analyzes the corresponding pricing optimization problem of two payper-use content providers. Section 3 models the case where the search engine exhibits a search bias, and computes the related equilibrium of the non-cooperative game played with each content provider. Section 4 compares the outputs obtained in both cases, neutral and non-neutral. Section 5 analyzes the incentives that the search engine and the content providers may have to integrate, and whether an integration is harmful for the users. Section 6 presents numerical results that are represented graphically and discussed in order to gain insight in the different trade-offs at stake. Section 7 presents an alternative model where the search engine post its price before each content provider does, and it discusses the differences between the models. And finally, some conclusions are drawn in Section 8.

\subsection{Related work}

Game theory was originally applied to the analysis of the interaction between economic agents, but more recently has extended towards the interaction between any kind of agents, specifically communications agents. This is the approach of the analysis and design of spectrum sharing [9] and rate adaptation [10] mechanisms in wireless radio networks. Furthermore, there are works that provide a mixed approach whereby both technical and economical decisions are modeled through game theory [11].

Our approach relates back to the analysis of the economical interactions. The analysis presented in this paper is in fact closely related to previous works on game 
theory-based models of network neutrality. Reference [12] studies the implications of two types of non-neutral behavior by a monopolistic ISP: charging side payments and multiple service classes provision. Reference [13] analyzes the case of two competitive ISPs and a single content provider, specifically the incentive that each ISP has in charging a side payment. Our work focuses also on the issue of side payment — charged by the search engine, instead of the ISP. However, departing from [13], in our work the search engine faces no competition from other search engines, while the content providers do. And, as we will see in the body of the paper, the search engine modifies the service that content providers receive when a side payment is charged, which is not the case in the network neutrality analysis in [12].

Our paper is to our knowledge one of the few ones dealing with a game-theory modeling and analysis of search neutrality. Reference [14] also addresses this research question. However, it focuses on the issue of content ranking - while our focus, as stated above, is on the issue of the side payment. Specifically, reference [14] compares different ranking behaviors of a search engine. In a neutral ranking, content is ranked based only on its relevance. The paper demonstrates, by means of specific examples, the incentives for the search engine to depart from this neutral behavior in two ways: a first one where the search engine gets a fraction of the add-generated revenue of the ranked content, and a second one where the search engine owns some of the ranked content.

Finally, there are other infomedation scenarios that are receiving increasing interest in the scientific community. As an example, a news aggregator may increase a reader's propensity to visit the content producer's site, but it may also deprive the latter of traffic that would otherwise flow to their sites [15]. Some conclusions and modeling elements might be reused across this scenario and our scenario-actually, Google plays a predominant role both as a search engine and as a news aggregator.

\section{GENERAL MODEL AND ANALYSIS OF THE NEUTRAL CASE}

The scenario modeled in this paper is shown in Fig. 1. It includes:

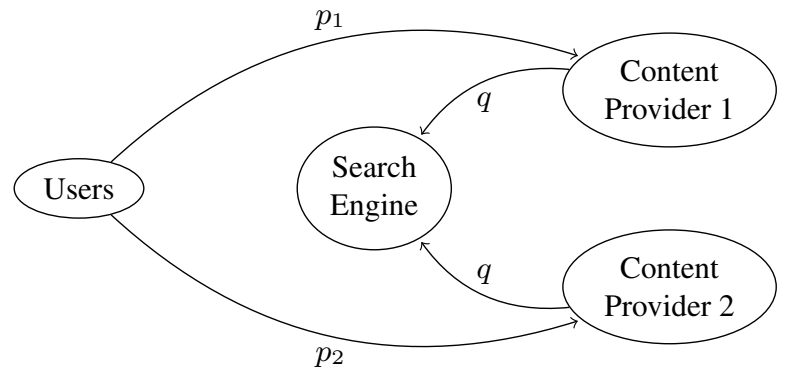

Figure 1. General model

- The users, who typically access the content stored at the content providers by using the search engine services.

- Two content providers (CP), which compete in providing paid content to the users, at prices $p_{i}(i=$ $1,2)$.

- The search engine (SE), which helps the users in locating the content at the content providers, and in the non-neutral case charges a price $q$ to each CP.

Arrows represent money flows, and are labeled by the corresponding unit price.

In this section, we model the case where there is no side payment from the CPs to the SE, that is, where $q=0$ in Fig. 1. We take this case as the baseline model, so that the search engine non-neutral behavior will be compared with this benchmark/baseline model.

The model builds on a previous contribution by the same authors, where a simpler game model was analyzed [16]. The model only covered the interaction between one $\mathrm{SE}$ and one $\mathrm{CP}$, providing a first approximation to the modeling of a side payment as a non-neutral behavior of a SE. By incorporating the competition between two content providers, this paper not only analyzes a more realistic model comprising an upstream monopoly (the SE) and a dowstream oligopoly (the two CPs), but in addition the extended model allows to analyze the richer relationships between the SE and each $\mathrm{CP}$ or both, as section 5 will show.

\subsection{Users}

We model the user preferences by means of a representative user who maximizes $U\left(x_{1}, x_{2}\right)-p_{1} x_{1}-p_{2} x_{2}$, where $x_{i}$ is the amount of content demanded from $\mathrm{CP}_{i}$ and $p_{i}$ is the price charged by $\mathrm{CP}_{i}$. The function $U$ is assumed 
to be quadratic and strictly concave:

$U\left(x_{1}, x_{2}\right)=\alpha_{1} x_{1}+\alpha_{2} x_{2}-\left(\beta_{1} x_{1}^{2}+2 \gamma x_{1} x_{2}+\beta_{2} x_{2}^{2}\right) / 2$

This expression is canonical in microeconomics for modeling the demand of differentiated products in a duopoly [17]. It is widely used in modeling user behavior for the following reasons. First, as it is shown below in (5), it produces the simplest downwards-sloping demand function, i.e. a linear one. Second, it models the demand for products supplied by two competing producers, where the products are not homogeneous; specifically, we assume here that $\gamma>0$, so that the products are substitutable, that is, a price increase in one product causes an increase in the demand of the other one-we will also contemplate that $\gamma=0$, so that the products are independent. The contents supplied by each provider are effectively substitutable goods. And third, it allows the model to abstract from the specific mechanisms that influence the behavior of the users, and to focus on other aspects, like the pricing and side payment of the content providers. The above expression for the utility has been widely used in telecommunications economics; see, for instance, how it has been used for modeling users demand for spectrum offered by oligopolistic wireless service providers in [18] and [19].

The above function can be expressed in matrix form as

$$
U(\boldsymbol{x})=\boldsymbol{\alpha}^{\prime} \boldsymbol{x}-\frac{1}{2} \boldsymbol{x}^{\prime} M \boldsymbol{x},
$$

where $\boldsymbol{x}=\left[\begin{array}{ll}x_{1} & x_{2}\end{array}\right]^{\prime}$, and $\boldsymbol{\alpha}=\left[\begin{array}{ll}\alpha_{1} & \alpha_{2}\end{array}\right]^{\prime}$ are column vectors, and

$$
M=\left[\begin{array}{ll}
\beta_{1} & \gamma \\
\gamma & \beta_{2}
\end{array}\right],
$$

with $\alpha_{i}, \beta_{i}, \beta_{1} \beta_{2}-\gamma^{2}, \alpha_{i} \beta_{j}-\alpha_{j} \gamma>0$, when $i, j=$ $1,2, \quad j \neq i$. These conditions ensure that the utility function is strictly concave, its global maximum is attained at a point of positive demands, and the utility is positive around the point of zero demands, $x_{1}=x_{2}=0$. We assume that the services provided by the CPs are substitutes or independent, so that $\gamma \geq 0$.

This utility function gives rise to a demand given by

$$
\boldsymbol{x}=\underset{\boldsymbol{a}}{\arg \max }\left[U(\boldsymbol{a})-\boldsymbol{p}^{\prime} \boldsymbol{a}\right]
$$

where $\boldsymbol{p}=\left[p_{1} p_{2}\right]^{\prime}$, which yields a linear dependence

$$
\begin{aligned}
& \boldsymbol{p}=\boldsymbol{\alpha}-M \boldsymbol{x}, \quad \text { and } \\
& \boldsymbol{x}=M^{-1}(\boldsymbol{\alpha}-\boldsymbol{p}),
\end{aligned}
$$

provided that quantities and prices are positive. We see that, first, $x_{1}$ (resp. $x_{2}$ ) is linearly decreasing in $p_{1}$ (resp. $p_{2}$ ) and linearly increasing in $p_{2}$ (resp. $p_{1}$ ); and second, the demand functions have the properties of symmetry of cross effects, that is,

$$
\frac{\partial x_{i}}{\partial p_{j}}=\frac{\partial x_{j}}{\partial p_{i}} .
$$

In words, the cross effect of a price rise of one service on the demand of the other one is the same as the reciprocal effect.

We can alternatively interpret the demand functions $x_{1}=D_{1}\left(p_{1}, p_{2}\right)$ and $x_{2}=D_{2}\left(p_{1}, p_{2}\right)$ as the total amount of users subscribing to each $\mathrm{CP}$, where $p_{1}$ (resp. $p_{2}$ ) would be the price charged by content provider 1 (resp. 2). We propose that

$$
\begin{aligned}
D_{1}\left(p_{1}, p_{2}\right) & =\eta_{0}\left(D_{01}-d_{1} p_{1}+c p_{2}\right), \\
D_{2}\left(p_{1}, p_{2}\right) & =\eta_{0}\left(D_{02}-d_{2} p_{2}+c p_{1}\right), \\
D\left(p_{1}, p_{2}\right) & =D_{1}\left(p_{1}, p_{2}\right)+D_{2}\left(p_{1}, p_{2}\right),
\end{aligned}
$$

where $\eta_{0}$ denotes the probability $\left(0<\eta_{0}<1\right)$ that the content is located by the search engine and therefore accessed by a user. $D_{0 i}$ is the maximum potential level of demand from content provider $i$, if all content was for free, and provided it was fully advertised by the search engine $\left(\eta_{0}=1\right)$. And $c$ is the same coefficient in both expressions due to the symmetry of cross effects. We assume that $p_{i}$ has a effect on the demand $D_{i}$ which is stronger than the cross effect produced produced by $p_{j}, j \neq i$; hence, $d_{i}>c$, which implies that $d_{1} d_{2}>c^{2}$. Note that the latter condition can be shown to be equivalent to $\beta_{1} \beta_{2}-\gamma^{2}>0$ that was stated above.

The value of $D_{0 i}-d_{i} p_{i}+c p_{j}$ can be interpreted as (being proportional to) the probability that a user, given the fact that he has "found" the content, actually subscribes to its service. That decision depends on the prices $p_{i}$ and $p_{j}$ set by the content providers, but also on the user's willingness-to-pay for the service.

The relationship between the two sets of parameters used to describe the demand is derived in Appendix A.1. 
Eqs. (7)-(8) can be rewritten in matrix form as

$$
\left[\begin{array}{l}
D_{1} \\
D_{2}
\end{array}\right]=\eta_{0}\left(\left[\begin{array}{l}
D_{01} \\
D_{02}
\end{array}\right]-S\left[\begin{array}{l}
p_{1} \\
p_{2}
\end{array}\right]\right)
$$

where

$$
S=\left[\begin{array}{cc}
d_{1} & -c \\
-c & d_{2}
\end{array}\right]
$$

\subsection{Content providers}

We assume, as described in Fig. 1, that $\mathrm{CP} i$ charges a fixed price $p_{i}$ to the users. In the baseline model the CPs are assumed not to incur costs, so that the CPs' profits are given by:

$$
\Pi_{c, i}\left(p_{1}, p_{2}\right)=p_{i} D_{i}, \quad i=1,2,
$$

Again, we assume that every piece of content that the users would like to access is stored by both content providers. However, the content will be located by the users by using the service of the search engine, which means that there is content stored at the $\mathrm{CP}$ which may end up not being accessed by the users if the search engine denies mentioning it.

\subsection{Search engine}

The role of the SE is to "help" users reach the content that is relevant to them.

We assume in our baseline model that the SE does not charge any usage-based price to the CPs. However, there is an additional revenue coming from sponsored links (displayed usually at the top and/or at the right of the regular/organic links), and which can reasonably be assumed to be proportional to the demand level. Indeed, users subscribing to a $\mathrm{CP}$ can be seen from the SE point of view as "satisfied" by the SE results, hence more likely to use that SE again and yield revenues through the sponsored links. Thus,

$$
\Pi_{s}=\lambda \cdot D
$$

with $\lambda>0$ the coefficient giving the revenue per subscriber to the CPs' service, coming from sponsored links. Therefore in the baseline model more demand at the $\mathrm{CP}$ level will give more revenue to the SE.

\subsection{Solution}

Given that the parameters $D_{0 i}, \eta_{0}, d_{i}, c$ and $\lambda$ are fixed, the only decision variables in this baseline model are the CPs' prices $p_{i}$. Each CP will charge a price $p_{i}^{(\mathrm{n})}$ so as to maximize its profits $\Pi_{c, i}$. The analysis framework is that of non-cooperative game theory [20], and the equilibrium concept that of Nash equilibrium, which is a strategy profile $\left(p_{1}^{(\mathrm{n})}, p_{2}^{(\mathrm{n})}\right)$ such that no player can unilaterally increase its profits through a price change.

Since the profit functions of the CPs $\left(\Pi_{c, i}\right)$ are clearly concave and continuously differentiable, the equilibrium is reached when the first order conditions (FOCs)

$$
\begin{gathered}
\frac{\partial \Pi_{c, 1}}{\partial p_{1}}=\eta_{0}\left(D_{01}-2 d_{1} p_{1}+c p_{2}\right)=0 \\
\frac{\partial \Pi_{c, 2}}{\partial p_{2}}=\eta_{0}\left(D_{02}-2 d_{2} p_{2}+c p_{1}\right)=0
\end{gathered}
$$

are simultaneously fulfilled, giving as solutions

$$
\left[\begin{array}{c}
p_{1}^{(\mathrm{n})} \\
p_{2}^{(\mathrm{n})}
\end{array}\right]=R\left[\begin{array}{c}
D_{01} \\
D_{02}
\end{array}\right]
$$

where

$$
R=\left[\begin{array}{cc}
2 d_{1} & -c \\
-c & 2 d_{2}
\end{array}\right]^{-1}
$$

Now, we define $D_{i}^{(n)}=D_{i}\left(p_{1}^{(\mathrm{n})}, p_{2}^{(\mathrm{n})}\right), \quad D^{(n)}=$ $D\left(p_{1}^{(\mathrm{n})}, p_{2}^{(\mathrm{n})}\right), \Pi_{c, i}^{(\mathrm{n})}=\Pi_{c, i}\left(p_{1}^{(\mathrm{n})}, p_{2}^{(\mathrm{n})}\right), \Pi_{s}^{(\mathrm{n})}=\Pi_{s}\left(p_{1}^{(\mathrm{n})}, p_{2}^{(\mathrm{n})}\right)$. After some algebra we obtain:

$$
\begin{aligned}
D_{i}^{(n)} & =\eta_{0} d_{i} p_{i}^{(\mathrm{n})}, \\
\Pi_{c, i}^{(\mathrm{n})} & =p_{i}^{(\mathrm{n})} D_{i}^{(n)}=\left(D_{i}^{(n)}\right)^{2} /\left(\eta_{0} d_{i}\right), \\
\Pi_{s}^{(\mathrm{n})} & =\lambda\left(D_{1}^{(n)}+D_{2}^{(n)}\right) .
\end{aligned}
$$

\section{NON-NEUTRAL MODEL}

The neutral case being solved, we now focus on the nonneutral behavior of a SE implementing side payments $q$ from each CP to the SE. The effect of the side payment is two-fold:

- Paying $q$ to the SE will increase the chances that $\mathrm{CP}_{i}$ 's content is located and accessed by the users, when users decide to use the SE. We model this effect through an increasing function $\eta_{1}(q)$. 
- The more the SE charges to the CP, the less the users trust the search results because the search bias will increase and the reputation of the SE will decrease, and the less likely they will use the SE. We model this reputation effect through a decreasing function $\eta_{2}(q)$.

As a consequence, the overall probability $\eta_{0}$ that a $\mathrm{CP}$ is seen by a user in the baseline model is proposed to be replaced here by a function

$$
\eta(q)=\eta_{1}(q) \eta_{2}(q)
$$

taking into account the positive and negative consequences of side payments commented above.

We will also assume

$$
\eta(0)=\eta_{0}
$$

so that a $\mathrm{CP}$ not paying any charge to the SE will reasonably be such that the model comes down to the baseline/neutral case. In the non-neutral case though, the SE could play with $\eta_{1}$ to impose $\eta(0)<\eta_{0}$ to enforce the $\mathrm{CP}$ to pay. This (other) case is left for further research; but would not add any major change to the analysis.

Taking into account the side payment, the demand functions $D_{i}$ and the profits $\Pi_{c, i}$ and $\Pi_{s}$ are now given by:

$$
\begin{gathered}
{\left[\begin{array}{c}
D_{1} \\
D_{2}
\end{array}\right]=\eta(q)\left(\left[\begin{array}{c}
D_{01} \\
D_{02}
\end{array}\right]-S\left[\begin{array}{l}
p_{1} \\
p_{2}
\end{array}\right]\right)} \\
\Pi_{c, i}\left(p_{1}, p_{2}, q\right)=\left(p_{i}-q\right) D_{i}, \quad i=1,2, \\
\Pi_{s}\left(p_{1}, p_{2}, q\right)=(q+\lambda)\left(D_{1}+D_{2}\right) .
\end{gathered}
$$

Note that these expressions generalize (10),(12), and (13). And that we also assume that the cross effects in $D_{1}$ and $D_{2}$ are symmetric, as stated in (6) for the neutral model.

The relationship between the two sets of parameters used to describe the demand for the non-neutral model is derived in Appendix A.2.

Some remarks follow with respect to the purpose and the scope of the non-neutral model presented in this section:

- The SE departs from the neutral behavior described in the previous section insofar as it modulates the content demand function by a function $\eta$ which is not only dependent on an objective ranking algorithm — as in the neutral case, but also on a side payment $q$.

- The side payment is assumed to be a tax levied by the SE in a non-discriminatory manner. Therefore, the SE is not assumed to play with a discriminatory $q_{i}$ in order to drive demand from one $\mathrm{CP}$ to the other one. In a similar fashion, no $\mathrm{CP}$ is assumed to choose a $q_{i}$ in order to drive demand from the competing $\mathrm{CP}$.

- The function $\eta(\cdot)$ therefore influences the overall demand faced by the two CPs. It aims to model a potential dead weight loss that the side payment may cause on the welfare of the operators and of the users. An interpretation may be that a low $\eta$ value would drive demand out from the CPs, while a high $\eta$ would drive demand back to the CPs.

\subsection{Solution}

Given $\eta(\cdot)$ and the parameters $D_{0 i}, d_{i}, c$ and $\lambda$, each of the two CPs and the SE interact strategically and noncooperatively in order to maximize their respective profits. That is, each CP will charge a price $p_{i}$ so as to maximize its profits $\Pi_{c, i}$, and simultaneously the SE will charge a side payment $q$ so as to maximize its profits $\Pi_{s}$. Again, we search for a Nash equilibrium $\left(p_{1}^{(\mathrm{nn})}, p_{2}^{(\mathrm{nn})}, q^{*}\right)$. Although we assume that prices are chosen simultaneously, what is relevant for the analysis is not the timing of the decisions but the fact that each player makes its choice without the knowledge of the other players' choices. The simultaneous-move assumption is commonplace in economic modeling, however an alternative assumption will be discussed in Section 7 .

Again, since the profit functions of the CPs $\left(\Pi_{c, i}\right)$ are concave and continuously differentiable, we search the equilibrium prices by solving the FOCs. Given a strategy $q$ of the SE, the FOCs can now be written as

$$
\eta(q)\left(\left[\begin{array}{l}
D_{01} \\
D_{02}
\end{array}\right]-R\left[\begin{array}{l}
p_{1} \\
p_{2}
\end{array}\right]+\left[\begin{array}{l}
d_{1} \\
d_{2}
\end{array}\right] q\right)=0
$$


And for the SE, differentiating with respect to $q$ and equating to zero, we get

$$
\begin{aligned}
\frac{\partial \Pi_{s}}{\partial q}=\left(D_{01}-\right. & \left.d_{1} p_{1}+c p_{2}+D_{02}-d_{2} p_{2}+c p_{1}\right) \\
& \times\left(\eta(q)+\eta^{\prime}(q)(q+\lambda)\right)=0 .
\end{aligned}
$$

We assume that either $D_{01}-d_{1} p_{1}+c p_{2}>0$ or $D_{02}-$ $d_{2} p_{2}+c p_{1}>0$, or both (otherwise the SE gets nothing). Then, $\frac{\partial \Pi_{s}}{\partial q}$ is of the same sign as $\eta(q)+\eta^{\prime}(q)(q+\lambda)$.

Additionally, the solution to (27), $q^{*}>0$, should lead to a better performance, in terms of probability for a $\mathrm{CP}$ to be visited, with respect to the neutral case, so that the non-neutral service is seen as an enhanced service for the CP. That is,

$$
\eta\left(q^{*}\right) \geq \eta(0)
$$

We propose the following expressions for $\eta_{1}(q)$ and $\eta_{2}(q)$ :

$$
\begin{aligned}
& \eta_{1}(q)=\left(1-\frac{1-\eta_{0}}{q+1}\right) \\
& \eta_{2}(q)=\frac{1}{q+1}
\end{aligned}
$$

These expressions comply with the desired behavior and assumptions, and they remain tractable in the analysis that is conducted hereafter. Specifically, first, $\eta_{1}(q)$ is increasing and $\eta_{2}(q)$ is decreasing. Second, the condition in (22) is satisfied. And, third, the requirement (28) is met as long as $\eta_{0}<1 / 2$ - taking into account the fact that $\eta_{1}(q) \eta_{2}(q)$ is decreasing when $q>1-2 \eta_{0}$. This may be interpreted as requiring that the visibility of the content in the neutral case be low enough.

Substituting (29) and (30) in (27), we get

$$
q^{*}=\frac{\eta_{0}+\lambda\left(1-2 \eta_{0}\right)}{\lambda-2+\eta_{0}}
$$

Requirement (28) becomes

$$
\begin{aligned}
\eta\left(q^{*}\right) & =\frac{\left(\lambda-2+\eta_{0}\right)\left(\lambda-\lambda \eta_{0}-\eta_{0}+\eta_{0}^{2}\right)}{4\left(\lambda+\eta_{0}-\lambda \eta_{0}-1\right)^{2}} \\
& =\frac{\left(\lambda-2+\eta_{0}\right)\left(\lambda-\eta_{0}\right)}{4\left(1-\eta_{0}\right)(\lambda-1)^{2}}>\eta_{0} .
\end{aligned}
$$

After some algebra and bearing in mind that $\eta_{0}<1 / 2$, the above inequality can be rewritten as

$$
\lambda^{2}-2 \lambda-\frac{\eta_{0}\left(2-3 \eta_{0}\right)}{\left(1-2 \eta_{0}\right)^{2}}>0
$$

which is satisfied if and only if

$$
\lambda>1+\sqrt{1+\frac{\eta_{0}\left(2-3 \eta_{0}\right)}{\left(1-2 \eta_{0}\right)^{2}}}=2+\frac{\eta_{0}}{1-2 \eta_{0}} .
$$

Analogously to the neutral case, $p_{1}^{(\mathrm{nn})}$ and $p_{2}^{(\mathrm{nn})}$ are the solutions of the FOCs (26) in the non-neutral case

$$
\left[\begin{array}{l}
p_{1}^{(\mathrm{nn})} \\
p_{2}^{(\mathrm{nn})}
\end{array}\right]=R\left(\left[\begin{array}{c}
D_{01} \\
D_{02}
\end{array}\right]+\left[\begin{array}{l}
d_{1} \\
d_{2}
\end{array}\right] q\right)
$$

Likewise, we introduce $D_{i}^{(\mathrm{nn})}=D_{i}\left(p_{1}^{(\mathrm{nn})}, p_{2}^{(\mathrm{nn})}, q^{*}\right)$, $D^{(\mathrm{nn})}=D\left(p_{1}^{(\mathrm{nn})}, p_{2}^{(\mathrm{nn})}, q^{*}\right), \quad \Pi_{c, i}^{(\mathrm{nn})}=\Pi_{c, i}\left(p_{1}^{(\mathrm{nn})}, p_{2}^{(\mathrm{nn})}, q^{*}\right)$ and $\quad \Pi_{s}^{(\mathrm{nn})}=\Pi_{s}\left(p_{1}^{(\mathrm{nn})}, p_{2}^{(\mathrm{nn})}, q^{*}\right)$. And we define $\eta^{*}=\eta\left(q^{*}\right)$. After some algebra we obtain:

$$
\begin{aligned}
& D_{1}^{(\mathrm{nn})}=\eta^{*} d_{1} p_{1}^{(\mathrm{n})}\left(1-q^{*} \frac{2 d_{1} d_{2}-c^{2}-c d_{2}}{2 d_{2} D_{01}+c D_{02}}\right), \\
& D_{2}^{(\mathrm{nn})}=\eta^{*} d_{2} p_{2}^{(\mathrm{n})}\left(1-q^{*} \frac{2 d_{1} d_{2}-c^{2}-c d_{1}}{2 d_{1} D_{02}+c D_{01}}\right), \\
& \Pi_{c, i}^{(\mathrm{n})}=\left(p_{i}^{(\mathrm{n})}-q^{*}\right) D_{i}^{(n)}=\left(D_{i}^{(\mathrm{nn})}\right)^{2} /\left(\eta^{*} d_{i}\right), \\
& \Pi_{s}^{(\mathrm{nn})}=\left(q^{*}+\lambda\right)\left(D_{1}^{(\mathrm{nn})}+D_{2}^{(\mathrm{nn})}\right) .
\end{aligned}
$$

It would remain to prove that, given $\left(p_{1}^{(\mathrm{nn})}, p_{2}^{(\mathrm{nn})}\right), q^{*}$ is a maximum of $\Pi_{s}$, which we do next. From (31) and (32) it follows that $q^{*}>0$. Besides,

$$
\begin{aligned}
& \frac{\partial^{2} \Pi_{s}}{\partial q^{2}}\left(p_{1}^{(\mathrm{nn})}, p_{2}^{(\mathrm{nn})}, q^{*}\right)= \\
& \quad-2 \frac{D_{1}^{(\mathrm{nn})}+D_{2}^{(\mathrm{nn})}}{\eta^{*}} \frac{\left(1-\eta_{o}\right)(\lambda-1)}{\left(1+q^{*}\right)^{4}}<0 .
\end{aligned}
$$

Thus, we conclude that $\Pi_{s}$ reaches a global maximum at $q=q^{*}$.

\section{COMPARISON BETWEEN THE NEUTRAL AND NON-NEUTRAL CASES}

In this section, the outcomes for the users, the CPs and the SE in the non-neutral case is compared with the baseline case, and constraints on the values of the parameters providing better output thanks to non-neutrality are derived. 


\subsection{Profits}

We first focus on the CPs profits $\Pi_{c, i}$, and the SE profits $\Pi_{s}$. Dealing with (18)-(20) and with (34)-(37), the following conditions are obtained:

Content providers:

$$
\begin{gathered}
\Pi_{c, 1}^{(\mathrm{nn})}>\Pi_{c, 1}^{(\mathrm{n})} \Longleftrightarrow \frac{2 d_{2} D_{01}+c D_{02}}{2 d_{1} d_{2}-c^{2}-c d_{2}}>\frac{q^{*}}{1-\sqrt{\frac{\eta_{0}}{\eta^{*}}}} \\
\Pi_{c, 2}^{(\mathrm{nn})}>\Pi_{c, 2}^{(\mathrm{n})} \Longleftrightarrow \frac{2 d_{1} D_{02}+c D_{01}}{2 d_{1} d_{2}-c^{2}-c d_{1}}>\frac{q^{*}}{1-\sqrt{\frac{\eta_{0}}{\eta^{*}}}}
\end{gathered}
$$

Search engine:

$$
\begin{aligned}
& \Pi_{s}^{(\mathrm{nn})}>\Pi_{s}^{(\mathrm{n})} \Longleftrightarrow \\
& \frac{d_{2}\left(2 d_{1}+c\right) D_{01}+d_{1}\left(2 d_{2}+c\right) D_{02}}{\left(d_{1}+d_{2}\right)\left(2 d_{1} d_{2}-c^{2}\right)-2 d_{1} d_{2} c}>\frac{q^{*}}{1-\frac{\lambda}{\lambda+q^{*}} \frac{\eta_{0}}{\eta^{*}}}
\end{aligned}
$$

In the rest of the section, we will consider the case in which both CPs have the same characteristics, so that expressions would allow to gain an insight. Notation can be simplified as $d_{1}=d_{2}=d$ and $D_{01}=D_{02}=D_{0}$. We have checked that the non-symmetric case does not add value to the conclusions.

For the neutral case,

$$
\begin{aligned}
p_{i}^{(\mathrm{n})} & =p^{(\mathrm{n})}=\frac{D_{0}}{2 d-c}, \\
D_{i}^{(n)} & =\eta_{0} d p^{(\mathrm{n})}, \\
\Pi_{c, i}^{(\mathrm{n})} & =\Pi_{c}^{(\mathrm{n})}=\left(D_{i}^{(n)}\right)^{2} /\left(\eta_{0} d\right), \\
\Pi_{s}^{(\mathrm{n})} & =2 \lambda D_{i}^{(n)} .
\end{aligned}
$$

And for the non-neutral case,

$$
\begin{aligned}
& p_{i}^{(\mathrm{nn})}=p^{(\mathrm{nn})}=p^{(\mathrm{n})}+\frac{d q^{*}}{2 d-c}=\frac{D_{0}+d q^{*}}{2 d-c}, \\
& D_{i}^{(\mathrm{nn})}=\eta^{*} d p^{(\mathrm{n})}\left(1-q^{*} \frac{d-c}{D_{0}}\right), \\
& \Pi_{c, i}^{(\mathrm{nn})}=\Pi_{c}^{(\mathrm{nn})}=\eta^{*} d\left(p^{(\mathrm{n})}\right)^{2}\left(1-q^{*} \frac{d-c}{D_{0}}\right)^{2}, \\
& \Pi_{s}^{(\mathrm{nn})}=2\left(\lambda+q^{*}\right) D_{i}^{(\mathrm{nn})} .
\end{aligned}
$$

Thus, the conditions for improvement under non-neutral behavior become the following ones:

$$
\begin{aligned}
\Pi_{c}^{(\mathrm{nn})}>\Pi_{c}^{(\mathrm{n})} & \Longleftrightarrow C 1 \equiv \frac{D_{0}}{d-c}>\frac{q^{*}}{1-\sqrt{\frac{\eta_{0}}{\eta^{*}}}},(50) \\
\Pi_{s}^{(\mathrm{nn})}>\Pi_{s}^{(\mathrm{n})} & \Longleftrightarrow \quad C 2 \equiv \frac{D_{0}}{d-c}>\frac{q^{*}}{1-\frac{\lambda}{\lambda+q^{*}} \frac{\eta_{0}}{\eta^{*}}} .
\end{aligned}
$$

On the minimum value of $\lambda$. In the following paragraphs, we derive the restrictions that $C 1$ imposes on $\lambda$.

Note that the right-hand side of the inequality in $C 1$ is decreasing in $\lambda$. This follows from the two facts:

- $\eta^{*}$ is increasing in $\lambda$

$$
\begin{aligned}
\eta^{*} & =\frac{\left(\lambda-2+\eta_{0}\right)\left(\lambda-\eta_{0}\right)}{4\left(1-\eta_{0}\right)(\lambda-1)^{2}} \\
& =\frac{1}{4\left(1-\eta_{0}\right)}\left(1-\frac{\left(1-\eta_{0}\right)^{2}}{(\lambda-1)^{2}}\right) .
\end{aligned}
$$

- $q^{*}$ is decreasing in $\lambda$

$$
\frac{\partial q^{*}}{\partial \lambda}=\frac{-2\left(1-\eta_{0}\right)^{2}}{\left(\lambda-2+\eta_{0}\right)^{2}}<0
$$

Therefore, for a given value of $D_{0} /(d-c)$, condition $C 1$ is equivalent to imposing a minimum value for $\lambda$. Furthermore, the lower $D_{0} /(d-c)$, the higher that minimum value will be. This may be interpreted as requiring that the $\mathrm{SE}$, in the absence of side payments, get a minimum revenue from the user demand for content. Conversely, for a given $\lambda, C 1$ gives a lower bound for $D_{0} /(d-c)$, and the higher $\lambda$, the less restrictive that lower bound is. Likewise, this may be interpreted as requiring that the users be willing to pay a minimum amount for the service.

\subsection{Consumer surplus}

Another outcome to compare is the consumer surplus, representing the aggregated value that users get from the whole service. We compute the consumer surplus as [17] [21]

$$
\mathrm{CS}(\boldsymbol{x})=U(\boldsymbol{x})-\boldsymbol{p}^{\prime} \boldsymbol{x},
$$

where again $\boldsymbol{x}=\left[\begin{array}{ll}D_{1} & D_{2}\end{array}\right]^{\prime}$ is the column vector of actual demands and $\boldsymbol{p}=\left[\begin{array}{ll}p_{1} & p_{2}\end{array}\right]^{\prime}$ that of the prices. The 
consumer surplus (aka. Marshallian consumer surplus) at the equilibrium price $p$ is computed as the integral of the demand curve between $p$ and the price which sets the demand to zero. When the expression of the utility is of the quasilinear type, as it is our case $\left(U\left(x_{1}, x_{2}\right)-\right.$ $\left.p_{1} x_{1}-p_{2} x_{2}\right)$, the consumer surplus ends up being equal to the value of the utility at the optimum quantities, and it gives a money-metric utility measure at these quantities. As such, the consumer surplus is an equivalent measure to the producer's profit.

Eq. (52) can be rewritten, using (2) and (4), as

$$
\mathrm{CS}(\boldsymbol{x})=\frac{1}{2} \boldsymbol{x}^{\prime} M \boldsymbol{x}, \quad \text { or }
$$

in the case of symmetric CPs, from (66) and (67),

$$
\begin{aligned}
\mathrm{CS}^{(\mathrm{n})} & =\frac{\left(D_{i}^{(n)}\right)^{2}}{\eta_{0}(d-c)}, \\
\mathrm{CS}^{(\mathrm{nn})} & =\frac{\left(D_{i}^{(\mathrm{nn})}\right)^{2}}{\eta^{*}(d-c)}
\end{aligned}
$$

Now, from (43) and (47) it easily follows that $\mathrm{CS}^{(\mathrm{nn})}>$ $\mathrm{CS}^{(\mathrm{n})}$ iff

$$
\mathrm{CS}^{(\mathrm{nn})}>\mathrm{CS}^{(\mathrm{n})} \Longleftrightarrow \frac{D_{0}}{d-c}>\frac{q^{*}}{1-\sqrt{\frac{\eta_{0}}{\eta^{*}}}},
$$

which is the same condition as in (50)

Finally, from (50), (51), and (56) it follows that, in the symmetric case,

$$
\Pi_{c}^{(\mathrm{nn})}>\Pi_{c}^{(\mathrm{n})} \Longleftrightarrow \mathrm{CS}^{(\mathrm{nn})}>\mathrm{CS}^{(\mathrm{n})} \Longrightarrow \Pi_{s}^{(\mathrm{nn})}>\Pi_{s}^{(\mathrm{n})} .
$$

\section{DOWNSTREAM INTEGRATION}

In the previous sections, the CPs are assumed to act independently from each other and from the SE. In this section, we analyze the scenarios that arise when the SE integrates downstream, that is, when the SE is integrated with one $\mathrm{CP}$ and when the SE is integrated with both CPs. We call these scenarios "vertical integration" and "industry integration", respectively.

The models analyzed in this section build on the non-neutral model presented in Section 3, and on the assumptions made there, that is, that the cross effects in $D_{1}$ and $D_{2}$ are symmetric.

\subsection{Vertical integration}

First, the case where the SE integrates with $\mathrm{CP}_{2}$ is analyzed. Given $\eta(\cdot)$ and the parameters $D_{0 i}, d_{i}, c$ and $\lambda, \mathrm{CP}_{1}$ and $\mathrm{SE}+\mathrm{CP}_{2}$ interact strategically and noncooperatively in order to maximize their respective profits. That is, $\mathrm{CP}_{1}$ will charge a price $p_{1}$ so as to maximize its profits $\Pi_{c, 1}$, and simultaneously $\mathrm{SE}+\mathrm{CP}_{2}$ will charge a side payment $q$ and a price $p_{2}$ so as to maximize its joint profit

$$
\Pi=\Pi_{s}+\Pi_{c, 2}=(\lambda+q) D_{1}+\left(\lambda+p_{2}\right) D_{2} .
$$

We search for a Nash equilibrium $\left(p_{1}^{(\mathrm{vi})}, p_{2}^{(\mathrm{vi})}, q^{(\mathrm{vi})}\right)$ by solving the optimization problems

$$
\left.\begin{array}{rl}
\max _{p_{1}} & \Pi_{c, 1} \\
\text { s.t. } & q \leq p_{1}, \\
& 0 \leq D_{01}-d_{1} p_{1}+c p_{2} .
\end{array}\right\}
$$

and

$$
\left.\begin{array}{ll}
\max _{p_{2}, q} \Pi & \\
\text { s.t. } & 0 \leq p_{2}, \\
& 0 \leq D_{02}+c p_{1}-d_{2} p_{2}, \\
& 0 \leq q .
\end{array}\right\}
$$

The detailed derivation of the solution is given in Appendix B.2.

\subsection{Industry integration}

The case where the SE integrates with both $\mathrm{CP}_{1}$ and $\mathrm{CP}_{2}$ is now analyzed. Given $\eta(\cdot)$ and the parameters $D_{0 i}, d_{i}, c$ and $\lambda$, the $\mathrm{SE}+\mathrm{CP}_{1}+\mathrm{CP}_{2}$ takes an optimal decision in order to maximize its joint profits. That is, the $\mathrm{SE}+\mathrm{CP}_{1}+\mathrm{CP}_{2}$ will choose a side payment $q$ and charge a couple of prices $p_{1}$ and $p_{2}$ so as to maximize its joint profit

$$
\begin{aligned}
\Pi=\Pi_{s}+\Pi_{c, 1}+\Pi_{c, 2}= \\
\quad\left(\lambda+p_{1}\right) D_{1}+\left(\lambda+p_{2}\right) D_{2} .
\end{aligned}
$$


We now search for the values $\left(p_{1}^{(\mathrm{ii})}, p_{2}^{\text {(ii) }}, q^{(\mathrm{ii})}\right)$ that are the solution to

$$
\left.\begin{array}{ll}
\max _{p_{1}, p_{2}, q} \Pi & \\
\text { s.t. } \quad & 0 \leq p_{1}, \\
& 0 \leq p_{2}, \\
& 0 \leq D_{01}-d_{1} p_{1}+c p_{2}, \\
& 0 \leq D_{02}+c p_{1}-d_{2} p_{2}, \\
& 0 \leq q .
\end{array}\right\}
$$

The detailed derivation of the solution is given in Appendix B.1.

\section{NUMERICAL RESULTS AND DISCUSSION}

In this section, the results derived above analytically are computed numerically and represented graphically in order to gain insight in the different tradeoffs at stake, and the results are discussed. In Section 6.1 the outcome for each stakeholder is computed for the neutral and nonneutral cases. Sections 6.2 and 6.3 analyze the incentives for integration in the vertical integration and industry integration cases, respectively.

\subsection{Neutral vs. non-neutral cases}

The outcome for each stakeholder is computed for the neutral and non-neutral cases, and the influence of each parameter is analyzed.

On the conditions $C 1$ and $C 2$. In Fig. 2, the domains such that conditions $C 1$ and $C 2$ hold are represented as a function of $\lambda$.

First, note that the graph represents the right-hand side of each inequality in (50) and (51) as a function of the parameter $\lambda$, and each pair of curves is generated with a different value for the parameter $\eta_{0}$. And second, the represented range of $\lambda$ complies with (32).

Note that $C 1$ is indeed the most constraining condition (as proved previously), since the threshold curve lies above that of $C 2$ for every value of $\eta_{0}$. Also the graph demonstrates that there is a wide range of values for $D_{0} /(d-c)$ and $\lambda$ such that $C 2$ holds but $C 1$ does not, i.e.,

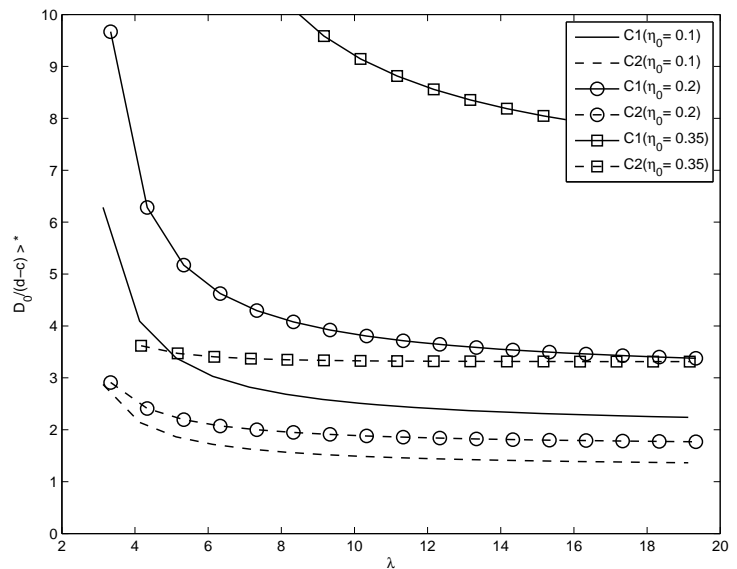

Figure 2. Thresholds for conditions $C 1$, and $C 2$ to hold, for $\eta_{0}=0.1,0.2,0.35$.

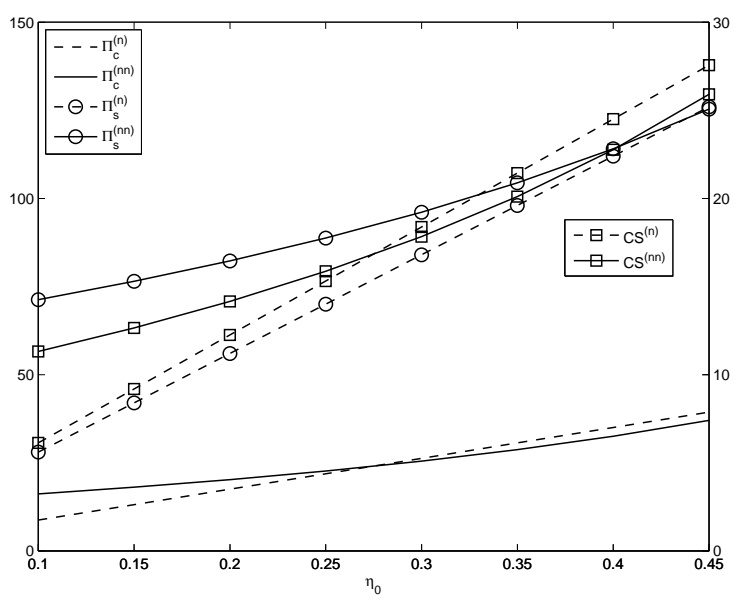

Figure 3. $\Pi_{c}, \Pi_{s}$ and CS for neutral and non-neutral cases $\left(D_{0} /(d-c)=6, \lambda=8\right)$

where the SE is better off but both the $\mathrm{CP}$ and the users are harmed with a non-neutral SE.

On the values of $\Pi_{c}, \Pi_{s}$ and CS. In Fig. 3, the values of $\Pi_{c}, \Pi_{s}$ and CS are represented as a function of $\eta_{0}$. The values of the parameters are $D_{0}=30, d=7, c=2$ and $\lambda=8$.

As expected from conditions $C 1$ and $C 2$ and the above discussion of Fig. 2, for low values of $\eta_{0}$, all stakeholders are better off in the non-neutral case. As $\eta_{0}$ increases, the first stakeholders to be harmed are the users and the CPs, while the SE remains as the only stakeholder to take profit from the search bias for high values of $\eta_{0}$. 


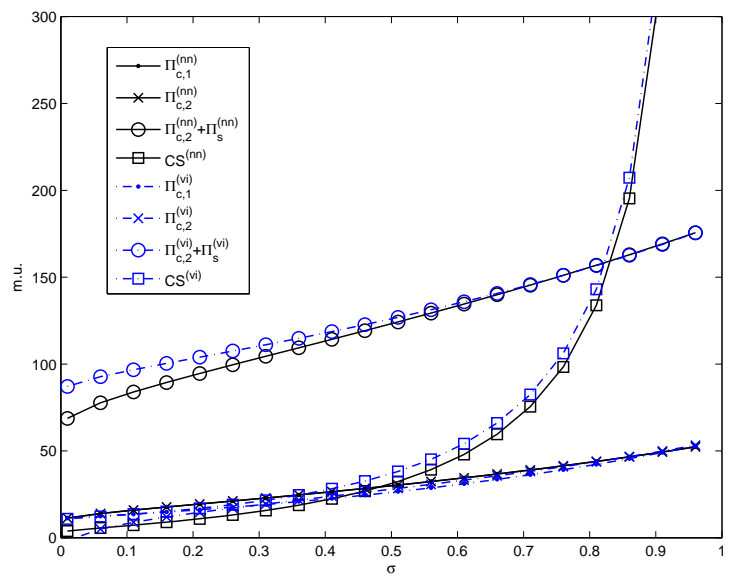

Figure 4. Profits and consumer surplus in non-neutral and vertical integration cases.

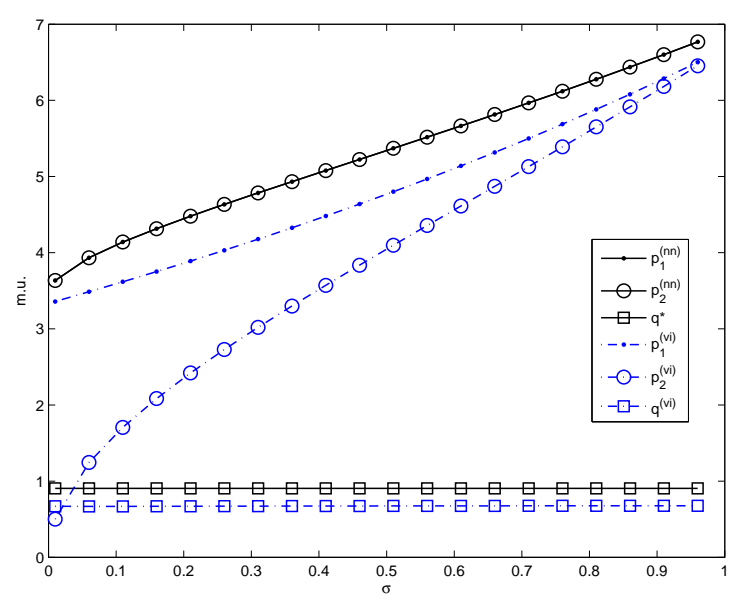

Figure 5. Prices and side payment in non-neutral and vertical integration cases.

\subsection{Vertical integration incentives}

We are interested in assessing whether both the SE and CP2 have any incentives to integrate, as modeled in section 5.1. In Figs. 4 and 5 the parameters take the following values: $D_{01}=D_{02}=30, d_{1}=d_{2}=5, \lambda=6$ and $\eta_{0}=0.2$. Following Section 3 , and specifically (67) in Appendix A.2, we define the degree of substitution $\sigma \triangleq \gamma^{2} /\left(\beta_{1} \beta_{2}\right)=c^{2} /\left(d_{1} d_{2}\right)$ [17], which measures how substitutable contents provided by $\mathrm{CP}_{1}$ and by $\mathrm{CP}_{2}$ are, ranging from 0 (independent contents) to 1 (perfect substitutes). In our setting, $c$ takes values between 0.5 and 4.9, so that $\sigma$ varies from 0.01 to 0.97 .

Fig. 4 shows the equilibrium profits of $\mathrm{SE}+\mathrm{CP}_{2}\left(\Pi_{s}^{(\mathrm{vi})}+\right.$ $\left.\Pi_{c, 2}^{(\mathrm{vi})}\right)$, covered in Section 5.1, and the sum of the equilibrium profits of $\mathrm{SE}$ and $\mathrm{CP}_{2}$ under the non-neutral case $\left(\Pi_{s}^{(\mathrm{nn})}+\Pi_{c, 2}^{(\mathrm{nn})}\right)$, covered in Section 3. For illustrative purposes, the profits of $\mathrm{CP}_{1}\left(\Pi_{c, 1}^{(\mathrm{nn})}\right.$ and $\left.\Pi_{c, 1}^{(\mathrm{vi})}\right)$ are also shown. Finally, the consumer surpluses for the non-neutral case and vertical integration $\left(\mathrm{CS}^{(\mathrm{nn})}\right.$ and $\left.\mathrm{CS}^{(\mathrm{vi})}\right)$ are also represented. Fig. 5 shows the equilibrium prices charged by the $\mathrm{CP}_{1}$ and the $\mathrm{CP}_{2}$ in the two cases $\left(p_{i}^{(\mathrm{nn})}\right.$ and $\left.p_{i}^{(\mathrm{vi})}\right)$. The equilibrium price charged by the $\operatorname{SE}\left(q^{*}\right.$ and $\left.q^{(\mathrm{vi})}\right)$ is also represented.

First, Fig. 4 shows that $\Pi_{s}^{(\mathrm{vi})}+\Pi_{c, 2}^{(\mathrm{vi})}$ is higher than $\Pi_{s}^{(\mathrm{nn})}+\Pi_{c, 2}^{(\mathrm{nn})}$. Additionally, as far as the situation of $\mathrm{CP}_{2}$ is concerned, although $\Pi_{c, 2}$ is reduced, $\mathrm{CP}_{1}$ gets a larger reduction in profits than $\mathrm{CP}_{2}$ when the vertical integration of $\mathrm{SE}$ and $\mathrm{CP}_{2}$ takes place. Second, Fig. 5 shows that all prices $p_{1}, p_{2}, q$ are reduced when $\mathrm{SE}$ and $\mathrm{CP}_{2}$ integrates. And that $\mathrm{CP}_{2}$ fixes a lower equilibrium price $p_{2}^{(\mathrm{vi})}$ than the corresponding $p_{1}^{(\mathrm{vi})}$. Third, as regards the users, Fig. 4 shows that $\mathrm{CS}^{(\mathrm{vi})}>\mathrm{CS}^{(\mathrm{nn})}$. And finally, as $\sigma$ increases, the profits difference between the two cases-but also the consumer surplus difference-gets smaller. We have checked that similar observations are obtained when $\lambda$ and $\eta_{0}$ are varied, and when $D_{01}, D_{02}, d_{1}$ and $d_{2}$ are varied independently, departing from the above symmetric scenario.

We may conclude that the $\mathrm{SE}$ and the $\mathrm{CP}_{2}$ have incentives to integrate. When the contents from each $\mathrm{CP}$ are close substitutes, these incentives get reduced, and in the limit, $\mathrm{SE}$ and $\mathrm{CP}_{2}$ are indifferent between integrating or not.

\subsection{Industry integration incentives}

We are now interested in assessing whether the SE and the two CPs have any incentives to integrate, as modeled in section 5.2. In Figs. 6 and 7 the parameters take the same values as in the previous subsection. Fig. 6 shows the overall profits $\left(\Pi_{s}+\Pi_{c, 1}+\Pi_{c, 2}\right)$ for the non-neutral $\left(\Pi^{(\mathrm{nn})}\right)$, vertical integration $\left(\Pi^{(\mathrm{vi})}\right)$ and industry integration $\left(\Pi^{(i i)}\right)$ cases. Accordingly, the consumer surplus in each of the three cases $\left(\mathrm{CS}^{(\mathrm{nn})}, \mathrm{CS}^{(\mathrm{vi})}, \mathrm{CS}^{(\mathrm{ii})}\right)$ is also represented. Fig. 7 shows the values for the prices charged by the $\mathrm{CP}_{1}$ and the $\mathrm{CP}_{2}$ in each case. The side payment charged by the $\mathrm{SE}$ is also represented.

First, Fig. 6 shows that, as expected, $\Pi^{(i i)}$ is greater than $\Pi^{(\mathrm{vi})}$ and $\Pi^{(\mathrm{nn})}$ (note that $\mathrm{SE}+\mathrm{CP}_{1}+\mathrm{CP}_{2}$ always has the option to choose the prices that match either $\Pi^{(\mathrm{vi})}$ or $\Pi^{(\mathrm{nn})}$ ). Additionally, the relationship between $\mathrm{CS}^{(\mathrm{nn})}, \mathrm{CS}^{(\mathrm{vi})}, \mathrm{CS}^{(\mathrm{ii})}$ 


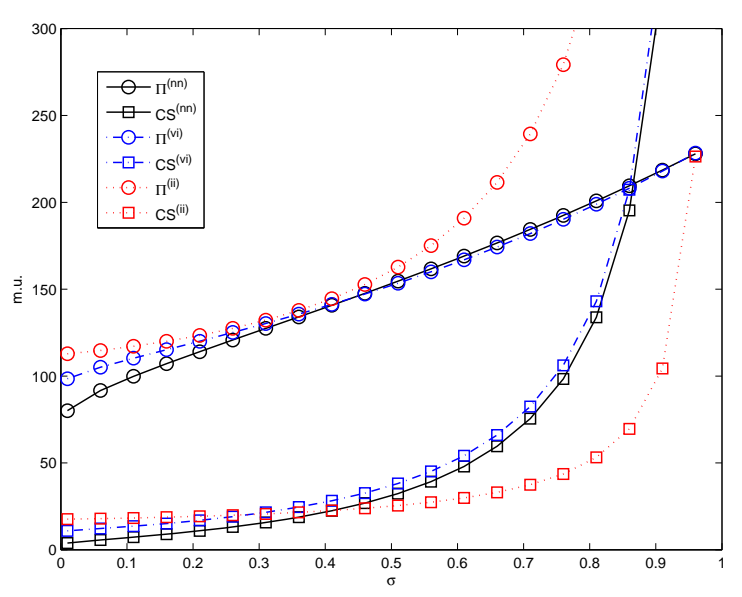

Figure 6. Profits and consumer surplus in non-neutral, vertical integration and industry integration cases.

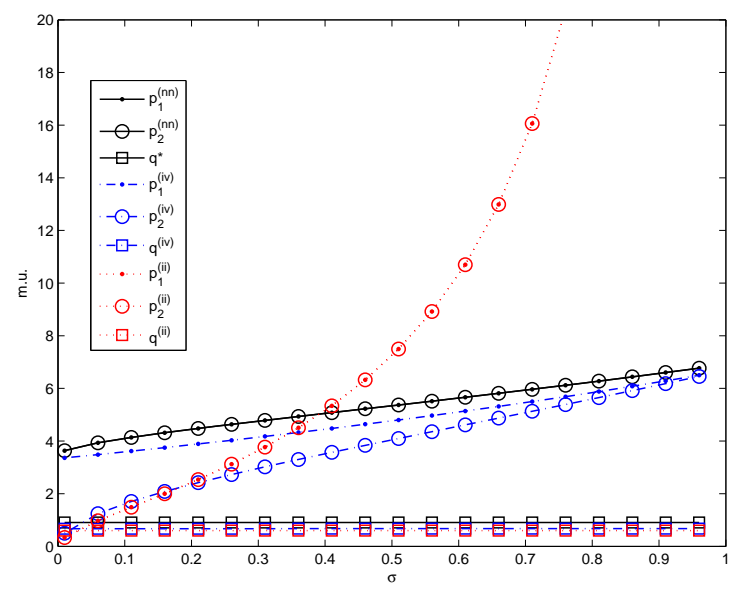

Figure 7. Prices and side payment in non-neutral, vertical integration and industry integration cases.

is different in each of the following three intervals:

$$
\left\{\begin{array}{l}
\mathrm{CS}^{(\mathrm{ii})}>\mathrm{CS}^{(\mathrm{vi})}>\mathrm{CS}^{(\mathrm{nn})} \text { if } \sigma<\sigma_{1}^{t h}, \\
\mathrm{CS}^{(\mathrm{vi})}>\mathrm{CS}^{(\mathrm{ii})}>\mathrm{CS}^{(\mathrm{nn})} \text { if } \sigma_{1}^{t h}<\sigma<\sigma_{2}^{t h} \\
\mathrm{CS}^{(\mathrm{vi})}>\mathrm{CS}^{(\mathrm{nn})}>\mathrm{CS}^{(\mathrm{ii})} \text { if } \sigma_{2}^{t h}<\sigma .
\end{array}\right.
$$

Furthermore, as $\sigma$ increases beyond $\sigma_{2}^{t h}$, the profits advantage in the industry integration case increases with respect to the other two cases. Analogously, the consumer surplus deterioration also increases beyond $\sigma_{2}^{t h}$. Second, Fig. 7 shows that the equilibrium price $p_{i}^{\text {(ii) }}$ charged by the CPs increases beyond the prices charged in the previous two cases.

We have also checked that similar observations are obtained when $\lambda$ and $\eta_{0}$ are varied, and when $D_{01}, D_{02}, d_{1}$ and $d_{2}$ are varied independently, departing from the above symmetric scenario.

We may conclude that the three operators have always an incentive to integrate. And that the incentives, for a degree of substitution $\sigma$ larger than a threshold, become very large. Note that when contents are close substitutes, the benefit that the operators get from cooperation-i.e., from avoiding competition-is greater than otherwise. On the other hand, the users will be worse off when contents are close substitutes.

\section{STACKELBERG MODEL}

In previous sections, we assumed that both CPs and the SE choose their respective prices in a simultaneous way. In this section, we model a situation where the SE chooses the side payment $q$, and the CPs, knowing this value, choose their respective prices $p_{1}$ and $p_{2}$. This game model is of a sequential-move type, it involves two stages and it is commonly known as Stackelberg model [20]. We argue that it is realistic that the SE, which is to some extent providing an upstream or wholesale service to the content providers, posts its price, and then the $\mathrm{CPs}$, which are providing a retail service to the users, post their respective prices with the knowledge of the SE's price.

In this section, we describe the Stackelberg model for the non-neutral cases and the vertical integration cases. Note that the Stackelberg model does not apply to neither the neutral case-since there is no side payment to postnor to the industry integration case-since there is only one player.

We have produced numerical results for the profits and the consumer surplus, using the same parameters as in Section 6, and we have checked that there is a negligible difference between the simultaneous-move and the Stackelberg models. Thus, no graphics is represented for the Stackelberg model.

\subsection{Stackelberg model for the non-neutral case}

As in Section 3, each of the two CPs and the SE interact strategically and non-cooperatively in order to maximize their respective profits. That is, each $\mathrm{CP}_{i}$ will charge a price $p_{i}$ so as to maximize its profits $\Pi_{c, i}$, and the SE will charge a side payment $q$ so as to maximize its profits $\Pi_{s}$. However, in this Stackelberg model, the SE posts the side 
payment $q$ in a first stage, and, in a second stage, each $\mathrm{CP}$ chooses simultaneously its respective price $p_{1}$ and $p_{2}$, with the knowledge of $q$. Formally, the equilibrium concept here is the subgame perfect Nash equilibrium [20]. We denote the equilibrium values $\left(p_{1}^{(\mathrm{nns})}, p_{2}^{(\mathrm{nns})}, q^{(\mathrm{nns})}\right)$.

The detailed derivation of the solution is given in Appendix B.3.

\subsection{Stackelberg model for the vertical integration case}

As in Section $6.2, \mathrm{CP}_{1}$ and $\mathrm{SE}+\mathrm{CP}_{2}$ interact strategically and non-cooperatively in order to maximize their respective profits. That is, $\mathrm{CP}_{1}$ will charge a price $p_{1}$ so as to maximize its profits $\Pi_{c, 1}$, and $\mathrm{SE}+\mathrm{CP}_{2}$ will charge a side payment $q$ and a price $p_{2}$ so as to maximize its joint profit $\Pi=\Pi_{s}+\Pi_{c, 2}$ However, in this Stackelberg model, the $\mathrm{SE}+\mathrm{CP}_{2}$ chooses the side payment $q$ in a first stage, and, in a second stage, $\mathrm{CP}_{1}$ chooses its price $p_{1}$ and simultaneously $\mathrm{SE}+\mathrm{CP}_{2}$ chooses $p_{2}$, with the knowledge of $q$. Again, the equilibrium concept here is the subgame perfect Nash equilibrium. We denote the equilibrium values $\left(p_{1}^{\text {(vis) }}, p_{2}^{\text {(vis) }}, q^{\text {(vis) }}\right)$.

The detailed derivation of the solution is given in Appendix B.4.

\section{CONCLUSIONS}

We have analyzed the interaction of a search engine and two content providers when the former exhibits a nonneutral behavior consisting in receiving a side payment in exchange for a search service improvement. We have also analyzed the incentives for the three operators to integrate, and the effect the integration would have on the users.

We have analyzed a model where the pricing decisions are taken simultaneously by every agent, that is, search engine and content providers. Additionally, we have analyzed an alternative model where the search engine posts first its side payment and then each one of the other agents, where applicable, chooses its price; and we have checked that the solutions differ in a negligible amount.

As regards the analysis of the non-neutral behavior, we conclude that under certain conditions, a side payment by the content provider to the search engine is beneficial for all stakeholders, since:
- the users are better off, which means that the improvement achieved by the increase in the likeliness to find the content compensates for the increase in the content price: more users "see" the content, and despite the fact that the price increases, the number of subscribers increases;

- the content providers benefit from a better visibility, allowing it to increase the subscription price to cover the payments to the search engine;

- and the search engine benefits from the increase in either the demand or the side payment, or in both.

Note that this win-win situation depends on the characteristics of the user demand, through the maximum tolerable price over the population; on the quality of the search activity; and on the fraction of SE profits that depends on the users demand but is independent of the side payment. As we have seen, for this situation to occur, it is required that:

- users be willing to pay a sufficient amount for the service,

- without side payments, the SE gets a sufficient revenue out of user demand for content,

- the visibility of the content in the neutral situation is low enough.

When this win-win situation is not achieved, the users and the CPs are the first stakeholders to suffer from non-neutral search activity.

The above results would support, absent this win-win situation, what the search engine neutrality advocates claim: that ex-ante regulatory measures should be put in place, i.e., side payments should not be allowed.

As regards the analysis of the different integration scenarios, we conclude that:

- There is an incentive for the SE and one $\mathrm{CP}$ to integrate; and the users would be better off if the integration took place.

- The SE and the two CPs are better off when they all integrate. However, when the contents provided by the CPs are close substitutes, the users are worse off in this situation, compared to the cases where the SE does not integrate and where the SE integrates to one $\mathrm{CP}$.

These last results would support that ex-post regulatory measures should be adopted so as to avoid the overall 
integration of the upstream SE and the downstream CPs. Actually, the removal of entry barriers and a non-discriminatory side payment-the one assumed throughout the paper-would suffice, since any attempt by the industry to integrate would be threatened by an entrant $\mathrm{CP}$ which would drive the industry integration scenario to one of vertical integration.

\section{ACKNOWLEDGEMENT}

This work has been supported by the Spanish Ministry of Economy and Competitiveness through project TIN201021378-C02-02

\section{A. RELATIONSHIP BETWEEN UTILITY AND DEMAND PARAMETERS}

\section{A.1. Neutral case}

The relationship between the parameter set $\alpha_{1}, \alpha_{2}, \beta_{1}, \beta_{2}$, $\gamma$, used in (1), and $\eta_{0}, D_{01}, D_{02}, c, d_{1}, d_{2}$ in (7)-(8) can be obtained from (5), as follows,

$$
S=\frac{1}{\eta_{0}} M^{-1}
$$

and

$$
\left[\begin{array}{l}
D_{01} \\
D_{02}
\end{array}\right]=\frac{1}{\eta_{0}} M^{-1} \boldsymbol{\alpha} .
$$

\section{A.2. Non-neutral case}

In a similar fashion, the relationship between the parameter set $\alpha_{1}, \alpha_{2}, \beta_{1}, \beta_{2}, \gamma$, used in (1), and $\eta(q), D_{01}, D_{02}, c, d_{1}, d_{2}$ in (23) can be obtained from (5), as follows,

$$
M^{-1}=\eta(q) S,
$$

and

$$
\left[\begin{array}{l}
D_{01} \\
D_{02}
\end{array}\right]=\frac{1}{\eta(q)} M^{-1} \boldsymbol{\alpha} .
$$

Note that $M$ depends on $q$, and thus it is different from the one in the neutral scenario.

\section{B. DETAILED SOLUTIONS}

\section{B.1. Industry integration Nash equilibrium}

Let us refer as $\mathcal{S}$ to the set defined by the constraints in (65). It is evident that $\Pi\left(p_{1}, p_{2}, q\right)$ is continuously differentiable on $\mathcal{S}$.

Also, it is easy to check that $\eta(q)$, and thus also $\Pi$ as a function of $q$, is decreasing for $q>1-2 \eta_{0}$. Therefore, we can choose a value $q^{u}>1-2 \eta_{0}$ such that if we add the restriction $q \leq q^{u}$ to those in (65) they define a compact set; let us refer to this set as $\mathcal{S}^{\prime}$.

Furthermore, if $\left(p_{1}, p_{2}, q\right) \in \mathcal{S} \backslash \mathcal{S}^{\prime}$ we have that $\Pi\left(p_{1}, p_{2}, q^{u}\right)>\Pi\left(p_{1}, p_{2}, q\right)$.

Consequently, the maximum of $\Pi$ on $\mathcal{S}^{\prime}$, which is guaranteed to exist, is also maximum of $\Pi$ on $\mathcal{S}$. Thus, the point $\left(p_{1}^{(\mathrm{ii})}, p_{2}^{(\mathrm{ii})}, q^{(\mathrm{ii})}\right)$ will be among those that satisfy the Karush-Kuhn-Tucker (KKT) conditions of the problem in (64)-(65).

We next present the solutions of the KKT conditions. First, let us define

$$
\begin{aligned}
\lambda_{1} & =\frac{d_{2} D_{01}+c D_{02}}{d_{1} d_{2}-c^{2}}, \\
\lambda_{2} & =\frac{c D_{01}+d_{1} D_{02}}{d_{1} d_{2}-c^{2}}, \\
\lambda_{1}^{\prime} & =\frac{D_{01}}{d_{1}-c}, \\
\lambda_{2}^{\prime} & =\frac{D_{02}}{d_{2}-c},
\end{aligned}
$$

and assume, without any loss of generality, that $\lambda_{1}^{\prime} \leq \lambda_{2}^{\prime}$. Then, it easily follows that $\lambda_{1}^{\prime} \leq \lambda_{1} \leq \lambda_{2} \leq \lambda_{2}^{\prime}$.

It can be shown there is only one point satisfying the KTT conditions, which is therefore the global maximum. Moreover, if the value of $\lambda$ is low enough (as long as condition (32) is met) the maximum is attained at an interior point: $p_{1}^{(\mathrm{ii})}>0, p_{2}^{(\mathrm{ii})}>0, q^{(\mathrm{ii})}>0$. Then, as $\lambda$ grows (while keeping the rest of the parameters constant) the values of $p_{1}^{(\mathrm{ii})}$ and $p_{2}^{\text {(ii) }}$ decrease until, first, $p_{1}=0$, and second, $p_{2}=0$. This behavior can be interpreted as follows. When $\lambda$ increases, it is more profitable to lower the prices charged by the content since this raises demand, and the increase in the profit from sponsored links outweighs the profit loss from content. In the limit, if $\lambda$ is high enough, it is preferable not to charge at all for the content and get all the profit from advertising.

The results obtained from the analysis of the KKT conditions can be summarized as follows: 
- if $\lambda<\lambda_{1}$ then $p_{1}^{(\mathrm{ii})}>0$ and $p_{2}^{(\mathrm{ii})}>0$

$$
\left[\begin{array}{c}
p_{1}^{(\mathrm{ii})} \\
p_{1}^{\text {(ii) }}
\end{array}\right]=\frac{1}{2} S^{-1}\left[\begin{array}{c}
D_{01} \\
D_{02}
\end{array}\right]-\frac{\lambda}{2}\left[\begin{array}{l}
1 \\
1
\end{array}\right]
$$

- if $\lambda_{1} \leq \lambda<\lambda_{2}^{\prime}$ then $p_{1}^{(\mathrm{ii})}=0$ and

$$
p_{2}^{\text {(ii) }}=\frac{D_{02}-\lambda\left(d_{2}-c\right)}{2 d_{2}}
$$

- if $\lambda_{2}^{\prime} \leq \lambda$ then $p_{1}^{(\mathrm{ii})}=p_{2}^{(\mathrm{ii})}=0$;

- for all values of $\lambda$,

$$
q^{(\mathrm{ii})}=1-2 \eta_{0} .
$$

\section{B.2. Vertical integration Nash equilibrium}

With regard to the maximization of $\Pi_{c, 1}$ (see (59)), it can be easily checked that, if the feasibility region is not empty, that is if

$$
D_{01}-d_{1} q+c p_{2} \geq 0,
$$

then the maximum is attained at

$$
p_{1}=\frac{1}{2}\left(\frac{D_{01}+c p_{2}}{d_{1}}+q\right) \text {. }
$$

Now we turn our attention to the maximization problem for $\Pi$ (see (61)).

We begin by noting that

$$
\lim _{q \rightarrow \infty} \Pi=D_{01}-d_{1} q+c p_{2}=\frac{D_{1}}{\eta(q)}
$$

and

$$
(\lambda+q) \eta(q)>1 \quad \text { if } \quad q>\frac{1-\lambda \eta_{0}}{\lambda+\eta_{0}-2} .
$$

By using these two observations, we can proceed in much the same way as we did in B.1 so as to show that $\Pi$ attains a global maximum in the feasibility region defined by (62); actually, in a compact subset of it.

From the analysis of the KKT conditions we obtained that there are two types of candidates to be the maximum: $\boldsymbol{y}^{(1)}=\left(p_{1}^{(1)}, p_{2}^{(1)}, q^{(1)}\right)$ and $\boldsymbol{y}^{(2)}=\left(p_{1}^{(2)}, 0, q^{(2)}\right)$. The first of them is an interior point, while for the second $p_{2}=0$. We now characterize each of the two solution types.
Solution $\boldsymbol{y}^{(1)}$. From

$$
\frac{\partial \Pi_{c, 1}}{\partial p_{1}}=\frac{\partial \Pi}{\partial p_{2}}=0
$$

it follows that

$$
\left[\begin{array}{l}
p_{1} \\
p_{2}
\end{array}\right]=R\left[\begin{array}{c}
d_{1} \\
c
\end{array}\right] q+R\left[\begin{array}{c}
D_{01} \\
D_{02}-\left(d_{2}-c\right) \lambda
\end{array}\right]
$$

or with a simplified notation,

$$
\left[\begin{array}{l}
p_{1} \\
p_{2}
\end{array}\right]=\left[\begin{array}{l}
A_{1} \\
A_{2}
\end{array}\right] q+\left[\begin{array}{l}
B_{1} \\
B_{2}
\end{array}\right]
$$

The definition of $A_{1}, A_{2}, B_{1}, B_{2}$ is easily obtained by comparing (76) and (77).

Substituting (77) into (23) yields

$$
\frac{1}{\eta(q)}\left[\begin{array}{c}
D_{1} \\
D_{2}
\end{array}\right]=\left[\begin{array}{c}
E_{1} \\
E_{2}
\end{array}\right] q+\left[\begin{array}{c}
F_{1} \\
F_{2}
\end{array}\right]
$$

where

$$
\left[\begin{array}{c}
E_{1} \\
E_{2}
\end{array}\right]=-S\left[\begin{array}{l}
A_{1} \\
A_{2}
\end{array}\right]
$$

and

$$
\left[\begin{array}{l}
F_{1} \\
F_{2}
\end{array}\right]=\left[\begin{array}{l}
D_{01} \\
D_{02}
\end{array}\right]-S\left[\begin{array}{l}
B_{1} \\
B_{2}
\end{array}\right]
$$

Now, from $\partial \Pi / \partial q=0$,

$$
\begin{aligned}
\left(\left(1-2 \eta_{0}\right) \lambda+\eta_{0}+\left(2-\eta_{0}-\lambda\right) q\right) \frac{D_{1}}{\eta(q)}+ & \\
\left(p_{2}+\lambda\right)\left(1-2 \eta_{0}-q\right) \frac{D_{2}}{\eta(q)} & =0,
\end{aligned}
$$

and substituting (77) and (78) into (81) gives the following cubic polynomial equation

$$
a_{3} q^{3}+a_{2} q^{2}+a_{1} q+a_{0}=0,
$$


where

$$
\begin{aligned}
a_{3}= & -A_{2} E_{2}, \\
a_{2}= & \left(2-\eta_{0}-\lambda\right) E_{1}+\left(B_{2}+\lambda\right) E_{2}+ \\
& \left(1-2 \eta_{0}\right) A_{2} E_{2}-A_{2} F_{2} \\
a_{1}= & \left(\lambda+\eta_{0}-2 \lambda \eta_{0}\right) E_{1}+\left(2-\eta_{0}-\lambda\right) F_{1}+ \\
& \left(1-2 \eta_{0}\right)\left(A_{2} F_{2}+B_{2} E_{2}+\lambda E_{2}\right)-\left(B_{2}+\lambda\right) F_{2},
\end{aligned}
$$

$a_{0}=\left(\lambda+\eta_{0}-2 \lambda \eta_{0}\right) F_{1}+\left(1-2 \eta_{0}\right)\left(B_{2}+\lambda\right) F_{2}$.

To get $\boldsymbol{y}^{(1)}$ we first obtain $q^{(1)}$ by solving numerically (82), which is then substituted into (77) to obtain $\left(p_{1}^{(1)}, p_{2}^{(1)}\right)$. Note that from the real roots of (82) we must discard those that do not give rise to a feasible solution. In other words, the obtained $\boldsymbol{y}^{(1)}$ must satisfy the constraints in (62) and (74).

Solution $\boldsymbol{y}^{(2)}$ In the same way as above, we obtain that

$$
\begin{aligned}
& p_{1}^{(2)}=\frac{1}{2}\left(\frac{D_{01}}{d_{1}}+q^{(2)}\right), \\
& p_{2}^{(2)}=0
\end{aligned}
$$

and $q^{(2)}$ is a root of

$$
b_{2} q^{2}+b_{1} q+b_{0}=0
$$

where

$$
\begin{aligned}
& b_{2}= d_{1}\left(\eta_{0}+\lambda-2\right)+\lambda, \\
& b_{1}= d_{1}\left(\lambda+\eta_{0}-2 \lambda \eta_{0}\right)+D_{01}\left(\eta_{0}+\lambda-2\right)+ \\
& \lambda\left(c \frac{D_{01}}{d_{1}}+2 D_{02}\right)-\lambda\left(1-2 \eta_{0}\right), \\
& b_{0}= D_{01}\left(\lambda+\eta_{0}-2 \lambda \eta_{0}\right)+ \\
& \lambda\left(1-2 \eta_{0}\right)\left(c \frac{D_{01}}{d_{1}}+2 D_{02}\right) .
\end{aligned}
$$

Now, we filter the roots of (89) by requiring $\boldsymbol{y}^{(2)}$ to satisfy

$$
\begin{aligned}
\frac{D_{01}}{d_{1}} & \geq q^{(2)}, \\
\lambda & >\frac{D_{02}+\frac{c}{2}\left(\frac{D_{01}}{d_{1}}+3 q^{(2)}\right)}{d_{2}-c} .
\end{aligned}
$$

The fact that if $\lambda$ exceeds a certain threshold the maximum for $\Pi$ is attained at point where $p_{2}$ has a similar interpretation to that given in Sect. B.1.

Although we have not been able to establish this mathematically, we conjecture that, for each set of values of the parameters, the KKT yield exactly one feasible candidate. That is, either there is one feasible solution of type $\boldsymbol{y}^{(1)}$ and none of type $\boldsymbol{y}^{(2)}$, or vice versa. This has been the case in all of our numerical experiments.

\section{B.3. Solution of the Stackelberg model for the non-neutral case}

The maximization problems faced by $\mathrm{CP}_{1}$ and $\mathrm{CP}_{2}$ have the same FOCs as in Section 3 (see (26)). From these we obtain $p_{1}(q)$ and $p_{2}(q)$, that is, the price $p_{1}$ (resp. $p_{2}$ ) that, for a given value of the side payment $q$, maximizes the profit of $\mathrm{CP}_{1}$ (resp. $\mathrm{CP}_{2}$ ). We then substitute $p_{1}(q)$ and $p_{2}(q)$ into the profit of the search engine, so that it becomes a function solely of the variable $q$

$$
\Pi_{s}(q)=\Pi_{s}\left(p_{1}(q), p_{2}(q), q\right) .
$$

The value $q^{\text {(nns) }}$ that maximizes $\Pi_{s}(q)$ is now sought by solving the FOC $\Pi_{s}^{\prime}(q)=0$, which after some algebra becomes the cubic polynomial equation

$$
q^{3}+3 q^{2}+a_{1} q+a_{0}=0
$$

where

$$
\begin{aligned}
& a_{1}=2 \lambda+2 \eta_{0}-\lambda \eta_{0}-b\left(\lambda+\eta_{0}-2\right) \\
& a_{0}=\lambda \eta_{0}+b\left(2 \lambda \eta_{0}-\lambda-\eta_{0}\right)
\end{aligned}
$$

and

$$
b=\frac{\left(2 d_{1}+c\right) d_{2} D_{01}+\left(2 d_{2}+c\right) d_{1} D_{02}}{2 d_{1} d_{2}\left(d_{1}+d_{2}-c\right)-c^{2}\left(d_{1}+d_{2}\right)} .
$$

We obtain $q^{\text {(nns) }}$ as the solution to (95) (after discarding the roots that do not give rise to feasible solutions). Then the prices charged by the CPs in the equilibrium are obtained as $p^{(\mathrm{nns})}=p_{1}\left(q^{(\mathrm{nns})}\right)$ and $p^{(\mathrm{nns})}=p_{2}\left(q^{(\mathrm{nns})}\right)$.

In this subsection we have restricted to derive the equations for the case in which the equilibrium is reached at an interior point. The conditions for this to occur and 
the equations for the other cases can be derived in a similar fashion as in B.1 and B.2.

\section{B.4. Solution of the Stackelberg model for the vertical integration case}

Here we proceed in much the same way as in Sect. B.3. From the FOCs we obtain the prices $p_{1}$ and $p_{2}$ as functions of the side payment $q$; the expressions for $p_{1}(q)$ and $p_{2}(q)$ are given in Eqs. (76) and (77). Then we substitute $p_{1}(q)$ and $p_{2}(q)$ into the profit of $\mathrm{SE}+\mathrm{CP}_{2}$, so that it becomes a function solely of the variable $q, \Pi(q)$. The value of $q^{\text {(vis) }}$ is then obtained by solving the FOC $\Pi^{\prime}(q)=0$, which becomes the cubic polynomial equation

$$
a_{3} q^{3}+a_{2} q^{2}+a_{1} q+a_{0}=0
$$

where

$$
\begin{gathered}
a_{3}=c A_{2}-d_{1}, \\
a_{2}=\lambda\left(c-d_{1}\right) B_{2}+\left(\eta_{0}+1\right)\left(c A_{2}-d_{1}\right)+ \\
\left(E_{1}+E_{2}\right)\left(2-\eta_{0}-\lambda\right), \\
a_{1}=\lambda\left(c-d_{1}\right)\left(\eta_{0}+1\right)+\eta_{0}\left(c A_{2}-d_{1}\right)+ \\
\left(E_{1}+E_{2}\right)\left(\eta_{0}+\lambda-2 \eta_{0} \lambda\right)+ \\
\left(F_{1}+F_{2}\right)\left(2-\eta_{0}-\lambda\right), \\
a_{0}=\eta_{0} \lambda\left(c-d_{1}\right) B_{2}+\left(F_{1}+F_{2}\right)\left(\eta_{0}+\lambda-2 \eta_{0} \lambda\right),
\end{gathered}
$$

and $E_{1}, E_{2}, F_{1}, F_{2}$ are given in Eqs. (77)-(80). Finally, $q^{\text {(vis) }}$ is the solution to (99) (after discarding the roots that do not give rise to feasible solutions), and then $p^{\text {(vis) }}=$ $p_{1}\left(q^{\text {(vis) }}\right)$ and $p^{(\mathrm{nns})}=p_{2}\left(q^{(\mathrm{vis})}\right)$.

As in the previous subsection, we have restricted to the case in which the equilibrium is reached at an interior point. The conditions for this to occur and the equations for the other cases can be derived in a similar fashion as in B.1 and B.2.

\section{AUTHORS' BIOGRAPHIES}

Luis Guijarro received the Ph.D. degree in Telecommunication from the Universitat Politècnica de València (UPV), Spain in 1998. He is an Associate Professor in Telecommunications Policy at the UPV. He researched in traffic management in ATM networks and in e-Government, and collaborated with the "Jean Monnet Chair on Telecommunications and Information Society Policy at the European Union", based in the UPV, and published the book "The Electronic Communications Policy of the European Union". His current research is focused on economic modeling of telecommunication service provision. He has contributed in the areas of $\mathrm{P} 2 \mathrm{P}$ interconnection, cognitive radio networks and search engine neutrality.

Vicent Pla received the M.E. and Ph.D. degrees in Telecommunication Engineering from the Universitat Politècnica de València (UPV), València, Spain. He is currently an Associate Professor with the Department of Communications at the UPV. He has published numerous papers in refereed journals and conference proceedings. His research interests include teletraffic and the modeling and performance evaluation of communication networks. During the past few years, most of his research activity has focused on resource management in wireless networks.

José-Ramón Vidal is currently an Associate Professor in Telematics at the Higher Technical School of Telecommunication Engineering of the Universitat Polit'ecnica de València (UPV), València, Spain. He obtained the $\mathrm{PhD}$ in Telecommunication Engineering from the UPV. His current research interest is focused on the area of application of game theory to resource allocation in cognitive radio networks. In these area he has published several papers in conference proceedings.

Jorge Martinez-Bauset received his Ph.D. in Telecommunication Engineering from the Universitat Politècnica de València (UPV), Spain, and currently is an associate professor at the same university. From 1987 to 1991 he was with QPSX Communications (Perth, Western Australia) participating in the design of the first IEEE 802.6 MAN. He has been with the Dep. of Communications of the UPV since 1991. He is a member of the Euro-NF Network of Excellence. He was recipient of the 1997s Alcatel Spain best Ph.D. Thesis award in access networks. His research interests are in the area of performance evaluation and traffic control for multiservice networks. In these areas he has published a number of papers in refereed journals and conference proceedings and has participated in different research projects sponsored by European and national governments, as well as by private companies. 


\section{REFERENCES}

1. European Commission. Antitrust: Commission Probes Allegations of Antitrust Violations by Google. Press Release 2010; URL http://europa. eu/rapid/pressReleasesAction.do? reference=IP /10/1624.

2. Pasquale III FA. Dominant Search Engines: An Essential Cultural \& Political Facility. SSRN eLibrary 2011; doi:10.2139/ssrn.1762241.

3. Viscusi W, Harrington J, Vernon J. Economics of regulation and antitrust. MIT Press Books 2005; 1.

4. Sterling G. Once Again Should Google be Allowed to Send Itself Traffic? Search Engine Land 2010; URL http://searchengineland. com/.

5. Manne GA, Wright JD. If Search Neutrality is the Answer, What's the Question? SSRN eLibrary 2011; doi:10.2139/ssrn.1807951.

6. Lenard T, May RE. Net Neutrality or Net Neutering: Should Broadband Internet Services be Regulated. Springer, 2006.

7. Hahn RW, Wallsten S. The economics of net neutrality. The Economists' Voice 2006; 3(6).

8. Altman E, Legout A, Xu Y. Network non-neutrality debate: An economic analysis. NETWORKING 2011. Springer, 2011; 68-81.

9. Ozel O, Uysal-Biyikoglu E. Network-wide energy efficiency in wireless networks with multiple access points. Transactions on Emerging Telecommunications Technologies 2012; :n/a-n/adoi:10.1002/ett. 2543. URL http://dx.doi.org/10.1002/ ett. 2543 .

10. Schmeink A. On fair rate adaption in interferencelimited systems. European Transactions on Telecommunications 2011; 22(5):200-210, doi:10.1002/ett. 1469. URL http://dx.doi.org/10.1002/ ett. 1469 .

11. Alptekin GI, Bener AB. Spectrum trading in cognitive radio networks with strict transmission power control. European Transactions on Telecommunications 2011; 22(6):282-295, doi:10.1002/ett. 1477. URL http://dx.doi.org/10.1002/ ett. 1477 .

12. Altman E, Bernhard P, Caron S, Kesidis G, RojasMora J, Wong S. A model of network neutrality with usage-based prices. Telecommunication Systems
2011; :1-9.

13. Coucheney P, Maillé P, Tuffin B. Impact of competition between isps on the net neutrality debate. Network and Service Management, IEEE Transactions on 2013; 10(4):1-9.

14. Coucheney P, Maillé P, Tuffin B. Comparison of Search Engines Non-Neutral and Neutral Behaviors. first Workshop on Pricing and Incentives in Networks (W-PIN 2012), co-located with ACM Sigmetrics/Performance, ACM, 2012; 1-8.

15. Palme E, Dellarocas C, Calin M, Sutanto J. Attention allocation in information-rich environments: the case of news aggregators. Proceedings of the 14th Annual International Conference on Electronic Commerce, ACM, 2012; 25-26.

16. Guijarro L, Pla V, Tuffin B, Maillé P, Coucheney P. A game theory-based analysis of search engine nonneutral behavior. Next Generation Internet (NGI), 2012 8th EURO-NGI Conference on, IEEE, 2012; 119-124.

17. Singh N, Vives $X$. Price and quantity competition in a differentiated duopoly. The RAND Journal of Economics 1984; :546-554.

18. Niyato D, Hossain E. Competitive pricing for spectrum sharing in cognitive radio networks: Dynamic game, inefficiency of nash equilibrium, and collusion. Selected Areas in Communications, IEEE Journal on 2008; 26(1):192-202.

19. Jia J, Zhang Q. Competitions and dynamics of duopoly wireless service providers in dynamic spectrum market. Proceedings of the 9th ACM international symposium on Mobile ad hoc networking and computing, ACM, 2008; 313-322.

20. Osborne M, Rubinstein A. A Course in Game theory. MIT Press, 1994.

21. Kluberg J, Perakis G. Generalized quantity competition for multiple products and loss of efficiency. Operations Research 2012; 60(2):335-350. 